\title{
Cardiac Magnetic Resonance and Amyloidosis: How can it Assist Clinical Reasoning?
}

\author{
Ibraim Masciarelli F. Pinto ${ }^{1,2}$ \\ Serviço de Imagem do Grupo Fleury, ${ }^{1}$ São Paulo, SP - Brazil \\ Serviço de Imagem do Instituto Dante Pazzanese, ${ }^{2}$ São Paulo, SP - Brazil
}

The real incidence of cardiac amyloidosis (CA) is still unknown, and this is, at least in part, due to difficulties in confirming diagnosis. Currently, however, different imaging exams and genetic tests can, in association with clinical presentation, confirm diagnosis. Cardiac magnetic resonance $(C M R)$ can contribute to confirmation of diagnosis and, more recently, to the screening of family members of carriers of hereditary forms of CA. ${ }^{1,2}$ In this article, we will review the contributions of $C M R$ in relation to clinical suspicion or screening for CA, initiating with a review of the information that the exam offers and, subsequently, discussing clinical scenarios in which CMR can be most useful.

\section{Evaluation of cardiac morphology}

CA leads to changes in cardiac structure. In the atria, dilatation and increased thickness of the septum usually occur, while, in more advanced phases, there may be slow flow and thrombi, which, when arrhythmias are present, may be difficult to identify via CMR. ${ }^{3}$

The ventricular myocardium usually shows increased thickness, typically at levels higher than those observed in patients with arterial hypertension; the levels are typically greater in forms of transthyretin amyloidosis than in light chain amyloidosis, and they may involve both ventricles. ${ }^{4,5}$

It is, moreover, not uncommon to find pericardial or pleural effusion, which are characteristics that can be easily seen on CMR. ${ }^{4,5}$

\section{Analysis of ventricular function}

CMR is considered the gold standard for analysis of global and regional ventricular contractility of both ventricles. ${ }^{6}$ Nevertheless, in clinical practice, analysis of ventricular contractility parameters is conducted, with good quality, using echocardiography, and resonance is reserved for conditions where there are doubts or conflicting echocardiograms. ${ }^{1}$ It is important to remember that ventricular contractility can be preserved for long

\section{Keywords}

Amyloidosis; Heart Failure; Ventricular Function.

Mailing Address: Ibraim Masciarelli F. Pinto •

Seção Médica de Tomografia e Ressonância Magnética. Av. Dr. Dante

Pazzanese, 500. Postal Code: 02404909, Vila Mariana, São Paulo, SP - Brazil.

E-mail: ibraim.pinto@grupofleury.com.br

Manuscript received September 07, 2021, revised manuscript September 24 2021, accepted September 24, 2021.

DOI: https://doi.org/10.36660/abchf.20210016 periods and that regional contractile analysis, especially based on strain analysis, can be more sensitive in detecting abnormalities in early stages of CA. ${ }^{1,4,5}$

Examples of morphological alterations in patients who are carriers of CA are displayed in Figure 1.

\section{Characterization of the myocardium - late enhancement techniques}

CMR with late enhancement (LE) is a well-established means of non-invasively investigating the existence of myocardial necrosis, inflammation, or fibrosis. Thanks to the dynamics of the material used as a contrast agent, a metal known as gadolinium, it is possible to identify the regions of the heart where it is retained and to quantify and define the morphological patterns of these regions. As these areas turn white and shiny, 7 to 12 minutes after the injection of the paramagnetic agent, this finding is called LE, which offers 2 types of information. ${ }^{5,6}$ The first is diagnostic in nature, given that different heart diseases tend to exhibit distinct LE patterns (Figure 2). In cases of CA, the most common pattern shows diffuse LE, often affecting the subendocardium or the entire transmural thickness of the heart walls, but different morphologies can arise, including involvement of the mesocardial segment and the pattern of focal enhancement. ${ }^{5,7}$

Characteristics of LE do not play a prominent role in defining the type of CA that patients present, but the presence and, in particular, the extent of LE are related to prognosis, and they add value to the markers that are classically used in clinical practice. Analyzing 250 patients, Fontana et al. demonstrated that LE quantification was an independent predictor of mortality, even considering variables such as pro-BNP, ejection fraction, and ventricular systolic volume index, diastolic function, and ventricular mass index. ${ }^{3,5}$

These elements make LE analysis a fundamental step in the evaluation of patients with clinical suspicion of CA.

\section{Characterization of the myocardium - T1 mapping}

Mapping, a more recently introduced form of characterizing the myocardium, comprises series based on changes that occur in T1, one of the parameters used to compose the CMR image. The data are presented in the form of quantifiable parametric maps, and they are able to reveal disease processes, even in conditions where LE is not observed, thus making them an important tool to complement diagnostic investigation of different heart diseases, including CA. ${ }^{4,5,8}$

Measurement of native T1 is conducted without the injection of contrast medium, which makes it an interesting alternative to examine patients who have contraindications 


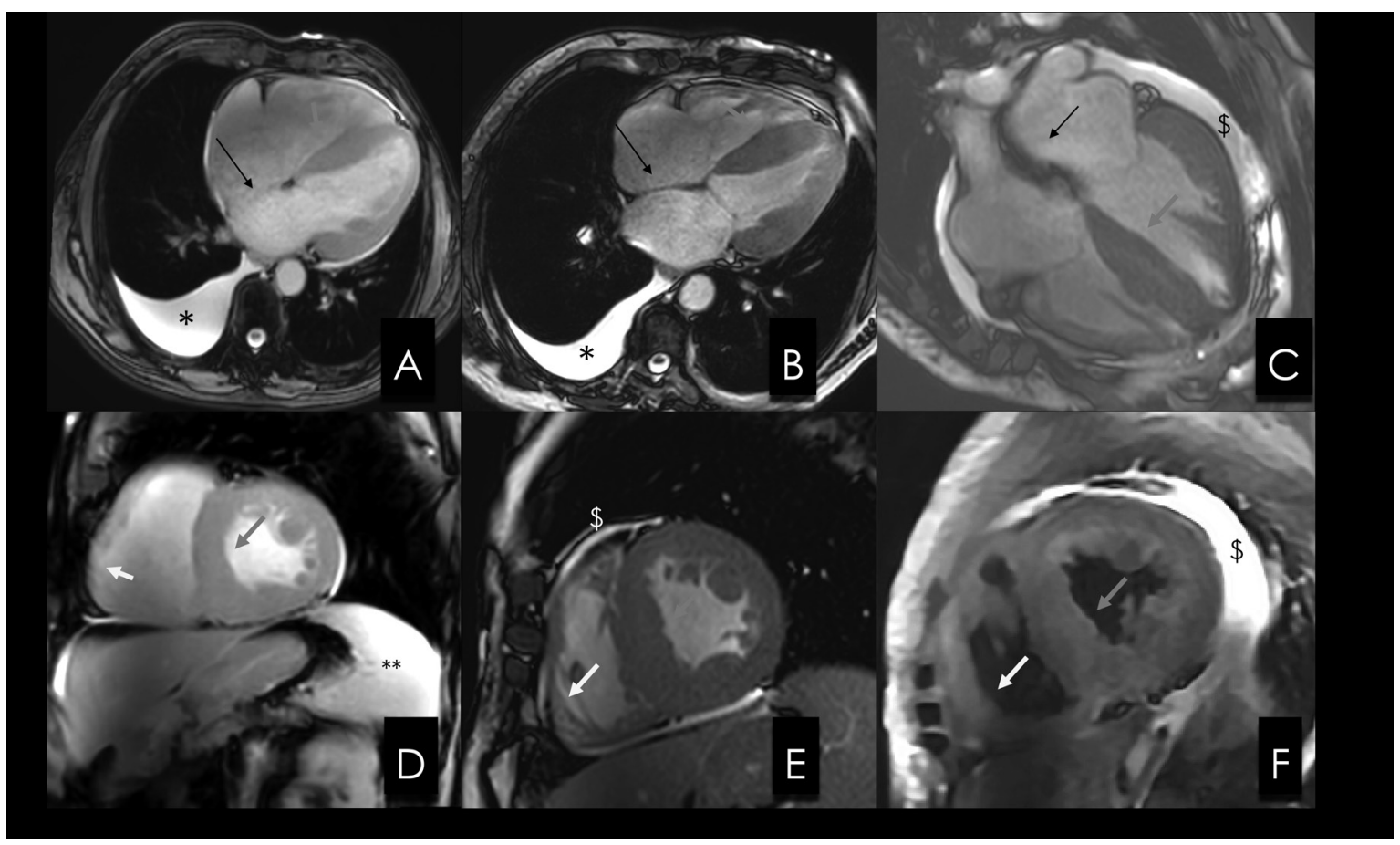

Figure 1 - CA can present different morphological patterns, depending on the degree of involvement and the duration of disease evolution. There is usually dilation of the atria $(1 A, 1 B$, and $1 C)$, while the thickness of the interatrial septum may be normal, slightly increased, or significantly increased, which is most characteristic ( $1 A, 1 B$, and $1 C$, respectively, black arrows). The thickness of the interventricular septum (red arrows) is usually increased, but the degree of involvement varies, as does the relationship between the measurements of the interventricular septum and the free wall. Right ventricle thickness may be preserved (yellow arrow, 1D), slightly enlarged (yellow arrow, 1E), or significantly enlarged (yellow arrow, 1F). Often, there are pericardial effusion $(1 \mathrm{C}$ and $1 F=\$)$, pleural effusion $(1 A$ and $1 B=*)$, and even ascites $(1 D=* *)$.

to the use of gadolinium, such as severe renal failure, or patients on a dialysis program. ${ }^{4,5,8}$ Diagnostic efficacy also increases in cases where the extracellular space is quantified; in this condition, a contrast agent is applied, and it reveals changes when there are processes that lead to expansion of the interstitium, which is the case with CA. ${ }^{4,5,8}$

The usefulness of this approach has been proven in a meta-analysis by Pan et al., who documented that the presence of elevated T1 mapping and extracellular space measurement led to improved diagnostic performance, compared to LE (odds ratio: $4.27 ; 95 \%$ confidence interval: $2.87-6.37$ vs. odds ratio: $2.60 ; 95 \%$ confidence interval: $1.90-3.56 ; p=0.03)$. However, the authors underscore that there may be cases where T1 mapping and the measurement of extracellular space do not confirm the diagnosis, and it may be necessary to evaluate the. ${ }^{8}$

\section{Contribution of resonance in different clinical scenarios}

\section{Screening family members for hereditary forms of CA}

Morphological and functional changes that result from CA may be useful for confirming diagnosis, but, as with LE, they may only appear later. What is expected in this scenario is that the diagnosis should be made earlier. The use of T1 mapping can be useful in this context, especially because, if this parameter is within the normal limits, it is very safe to rule out diagnosis of CA. ${ }^{4,5,8}$ Even though this approach is not widely used, it has shown great clinical potential.

\section{Differential diagnosis of myocardium with increased} thickness

Different heart diseases can cause increased thickness in the ventricular walls, and these conditions are often difficult to differentiate clinically. ${ }^{1,2,6,9}$ CMR can assist in the characterization of the etiology by identifying cases that are secondary to hypertension, cases of hypertrophic cardiomyopathy, Fabry disease, and CA. In this scenario, it is important to use all the tools that the exam provides, especially because both LE patterns and T1 mapping values vary according to etiology, and they can provide information that is essential to diagnosis. In the event that CA is confirmed, CMR results will make it possible to proceed to define the type of alteration and establish appropriate treatment as quickly as possible. ${ }^{1,2,6,9}$

Evaluation of patients with heart failure with preserved ejection fraction

In our experience, there have been cases of CA that have manifested as heart failure with preserved ejection fraction, even before there were significant increases in myocardial thickness. 


\section{Viewpoint}

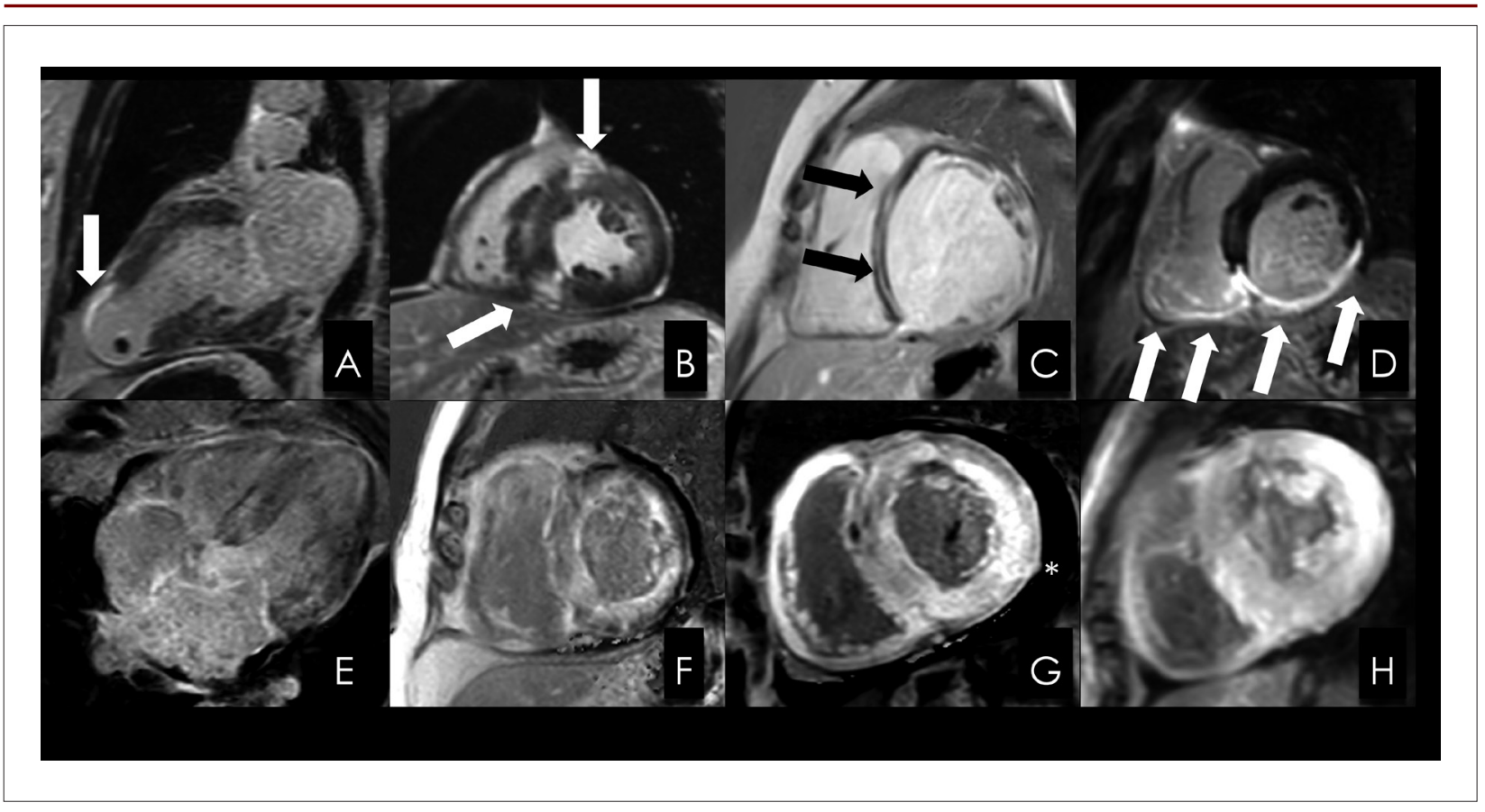

Figure 2 - LE patterns (arrows, 2A, 2B, 2C, and 2D) can help in diagnosis and provide prognostic data. Cases of Chagas disease show apical enhancement, at times accompanied by filling failure, as in $2 A$; the presence of increased mural thickness with $L E$ in the mesocardium at the meeting points of the right and left ventricles is characteristic of hypertrophic cardiomyopathy (2B). Cases of dilated cardiomyopathy often show areas of mesocardial LE in the interventricular septum (2C), and cases of coronary disease usually show enhancement in areas that are irrigated by only one coronary artery and have a "wave" morphology involving subendocardial to transmural regions. In cases of CA, LE is usually diffuse, and it is more relevant to the subendocardial region (2E and $2 F)$. In more advanced cases of the disease, the involvement becomes transmural ( $2 \mathrm{G}$ and $2 \mathrm{H})$. Due to difficulties in adjusting parameters that define $L E$, it can be difficult to visualize alterations, such as pericardial effusion $\left(2 G=^{*}\right)$, in these series. Studies have shown that cases of diffuse, transmural, extensive $L E$ are associated with worse prognosis $(2 H)(3)$

In this scenario as well, CMR can assist in identifying CA as the cause of the alterations and guide treatment. ${ }^{1,6}$

\section{Diagnostic uncertainty on other exams}

Due to the reduced intra- and interobserver variability and the superior capacity for myocardial characterization, CMR can be useful for defining diagnosis in cases where there are doubts after other forms of analysis, such as echocardiography, have been carried out. ${ }^{1,2,10}$

\section{In elderly patients with aortic stenosis}

The incidence of both aortic stenosis and CA increase as age advances, and this association is not rare; there are reports that both coexist in $13.9 \%$ to $16 \%$ of cases of aortic stenosis that will undergo percutaneous treatment. ${ }^{11} \mathrm{CMR}$ is recommended to clarify whether or not CA is present, especially if there is a disproportionate increase in myocardial thickness. LE techniques can identify myocardial injury and differentiate whether it is the consequence of valve damage or infiltrative disease. ${ }^{12}$ Furthermore, T1 mapping and increased extracellular space also favor diagnosis of infiltrative cardiomyopathy, and they may assist in the diagnostic strategy.

\section{Prognosis and treatment monitoring}

In addition to aiding in diagnosis of CA, CMR can also be useful in estimating the prognosis of patients with CA.
Although the applications of T1 mapping and estimation of extracellular space have the potential to contribute to this area, the main information regarding evolution is provided by LE. Fontana et al. demonstrated that the presence of LE is associated with worse evolution and that cases with transmural LE show more adverse events than cases with subendocardial LE. ${ }^{3}$ These data can help define the severity of the case and the treatment planning.

T1 mapping and extracellular space, on the other hand, have shown potential to monitor the therapeutic response in patients with specific treatments and, thus, assist in guiding the management strategy. ${ }^{4,5,8}$

Table 1 summarizes the potential contributions of CMR in different clinical scenarios.

\section{Conclusion}

CMR is highly useful in the evaluation of CA, and its application can have a positive impact on the management of these patients.

\section{Author contributions}

Conception and design of the research, Acquisition of data, Analysis and interpretation of the data, Writing of the manuscript and Critical revision of the manuscript for intellectual content: Pinto IMF. 
Viewpoint

Table 1 - Contribution of CMR to different clinical situations of patients with CA

\begin{tabular}{lr}
\hline Clinical scenario & Contribution of CMR \\
\hline Screening family members for hereditary forms of CA & $\begin{array}{r}\text { Early diagnosis of heart disease, preceding installation of structural heart } \\
\text { disease, especially with use of T1 mapping }\end{array}$ \\
\hline Differential diagnosis of myocardium with increased thickness & $\begin{array}{r}\text { Differential diagnosis based on use of T1 mapping and LE patterns, } \\
\text { contributing to early diagnosis and treatment }\end{array}$ \\
\hline Evaluation of patients with heart failure with preserved ejection fraction & Confirming diagnosis of CA and guiding correct treatment \\
\hline Diagnostic uncertainty on other exams & Confirming diagnosis and facilitating treatment \\
\hline In elderly patients with aortic stenosis & $\begin{array}{r}\text { Identification of cases of CA, based on analysis of T1 mapping and LE } \\
\text { pattern }\end{array}$ \\
\hline Prognosis and treatment monitoring & $\begin{array}{c}\text { The characteristics and extent of LE have an important prognostic impact. } \\
\text { T1 mapping is useful for evaluating the efficacy of specific treatment. }\end{array}$
\end{tabular}

CA: cardiac amyloidosis; CMR: cardiac magnetic resonance; LE: late enhancement

\section{Potential Conflict of Interest}

No potential conflict of interest relevant to this article was reported.

\section{Sources of Funding}

There were no external funding sources for this study.

\section{References}

1. Kittleson MM, Maurer MS, Ambardekar AV, Bullock-Palmer RP, Chang PP, Eisen HJ, et al. Cardiac Amyloidosis: Evolving Diagnosis and Management: A Scientific Statement from the American Heart Association. Circulation. 2020;142(1):7-22. doi: 10.1161/ CIR.0000000000000792.

2. Wang TKM, Abou Hassan OK, Jaber W, Xu B. Multi-Modality Imaging of Cardiac Amyloidosis: Contemporary Update. World J Radiol. 2020;12(6):87-100. doi: 10.4329/wjr.v12.i6.87.

3. Fontana M, Pica S, Reant P, Abdel-Gadir A, Treibel TA, Banypersad SM, et al. Prognostic Value of Late Gadolinium Enhancement Cardiovascular Magnetic Resonance in Cardiac Amyloidosis. Circulation. 2015:132(16):1570-9. doi: 10.1161/CIRCULATIONAHA.115.016567.

4. Baggiano A, Boldrini M, Martinez-Naharro A, Kotecha T, Petrie A, Rezk $\mathrm{T}$, et al. Noncontrast Magnetic Resonance for the Diagnosis of Cardiac Amyloidosis. JACC Cardiovasc Imaging. 2020;13(1):69-80. doi: 10.1016/j.jcmg.2019.03.026.

5. Jurcuţ R, Onciul S, Adam R, Stan C, Coriu D, Rapezzi C, et al. Multimodality Imaging in Cardiac Amyloidosis: A Primer for Cardiologists. Eur Heart J Cardiovasc Imaging. 2020;21(8):833-44. doi: 10.1093/ehjci/jeaa063.

6. Sara L, Szarf G, Tachibana A, Shiozaki AA, Villa AV, Oliveira AC, et al. II Diretriz de Ressonância Magnética e Tomografia Computadorizada Cardiovascular da Sociedade Brasileira de Cardiologia e do Colégio Brasileiro de Radiologia. Arq Bras Cardiol. 2014;103(6 Suppl 3):1-86. doi: 10.5935/abc.2014S006

\section{Study Association}

This study is not associated with any thesis or dissertation work.

\section{Ethics approval and consent to participate}

This article does not contain any studies with human participants or animals performed by any of the authors.

7. Zhao L, Tian Z, Fang Q. Diagnostic Accuracy of Cardiovascular Magnetic Resonance for Patients with Suspected Cardiac Amyloidosis: A Systematic Review and Meta-Analysis. BMC Cardiovasc Disord. 2016;16:129. doi: 10.1186/s12872-016-0311-6.

8. Pan JA, Kerwin MJ, Salerno M. Native T1 Mapping, Extracellular Volume Mapping, and Late Gadolinium Enhancement in Cardiac Amyloidosis: A Meta-Analysis. JACC Cardiovasc Imaging. 2020;13(6):1299-310. doi: 10.1016/j.jcmg.2020.03.010.

9. Martinez-Naharro A, Treibel TA, Abdel-Gadir A, Bulluck H, Zumbo G, Knight DS, et al. Magnetic Resonance in Transthyretin Cardiac Amyloidosis. J Am Coll Cardiol. 2017;70(4):466-77. doi: 10.1016/j. jacc. 2017.05.053

10. Maurer MS, Bokhari S, Damy T, Dorbala S, Drachman BM, Fontana $M$, et al. Expert Consensus Recommendations for the Suspicion and Diagnosis of Transthyretin Cardiac Amyloidosis. Circ Heart Fail. 2019;12(9):e006075. doi: 10.1161/CIRCHEARTFAILURE.119.006075

11. Castaño A, Narotsky DL, Hamid N, Khalique OK, Morgenstern R, DeLuca A, et al. Unveiling Transthyretin Cardiac Amyloidosis and its Predictors Among Elderly Patients with Severe Aortic Stenosis Undergoing Transcatheter Aortic Valve Replacement. Eur HeartJ. 2017;38(38):2879-87. doi: 10.1093/eurheartj/ehx350.

12. Bohbot Y, Renard C, Manrique A, Levy F, Maréchaux S, Gerber BL, et al. Usefulness of Cardiac Magnetic Resonance Imaging in Aortic Stenosis. Circ Cardiovasc Imaging. 2020;13(5):e010356. doi: 10.1161/ CIRCIMAGING.119.010356. 
